\title{
miR-128-3p inhibits glioma cell proliferation and differentiation by targeting NPTX1 through IRS-1/PI3K/AKT signaling pathway
}

\author{
LEIMING HUO $^{1}$, BIN WANG ${ }^{2}$, MAOHUA ZHENG $^{1}$, YONGHONG ZHANG $^{1}$, \\ JIGUANG XU $^{1}$, GANG YANG ${ }^{1}$ and QUANLIN GUAN ${ }^{1}$ \\ ${ }^{1}$ Department of Neurosurgery, The First Hospital of Lanzhou University, Lanzhou, Gansu 730000; \\ ${ }^{2}$ Department of Neurosurgery, The First People's Hospital of Longxi County, Dingxi, Gansu 730050, P.R. China
}

Received March 21, 2017; Accepted November 3, 2017

DOI: $10.3892 /$ etm.2019.7284

\begin{abstract}
It has been reported that glioma has a higher morbidity and mortality than other types of malignant brain tumor. While glioma has been extensively researched, the exact molecular mechanisms of its genesis and progression have remained to be fully elucidated. In order to explore a novel glioma-associated pathway which may represent a therapeutic target, 61 pairs of tumor tissues and adjacent normal tissues of glioma patients were collected and subjected to reverse-transcription quantitative polymerase chain reaction analysis, indicating that the relative expression of microRNA (miR)-128-3p was significantly decreased in the tumor tissues. However, the expression of neuronal pentraxin 1 (NPTX1) was obviously elevated. Through a bioinformatics analysis using Targetscan and transfection experiments, it was confirmed that NPTX1 was targeted by miR-128-3p. In the U251 human glioma cell line, transfection with miR-128-3p mimics increased the levels of phosphorylated insulin receptor substrate 1 (p-IRS-1), phosphoinositide-3 kinase (PI3K) and $\mathrm{p}-\mathrm{AKT}$, as demonstrated by western blot analysis. In addition, the proliferation rate of the cells was notably decreased following transfection with miR-128-3p mimics. Conversely, transfection with miR-128-3p inhibitor significantly increased the levels of p-IRS-1, PI3K and p-AKT, accompanied by an elevated proliferation rate of the cells. Therefore, it was indicated that miR-128-3p could reversely regulate NPTX1 expression. After the expression of NPTX1 was inhibited with specific small interfering RNA, the levels of p-IRS-1, PI3K and $\mathrm{p}-\mathrm{AKT}$ were obviously decreased, while the expression of miR-128-3p was not significantly changed. Overall, it was concluded that miR-128-3p suppresses glioma through the NPTX1/IRS-1/PI3K/AKT signaling pathway.
\end{abstract}

Correspondence to: Professor Quanlin Guan, Department of Neurosurgery, The First Hospital of Lanzhou University, 1 Donggang West Road, Lanzhou, Gansu 730000, P.R. China

E-mail: guanquanlin_jie@163.com

Key words: microRNA-128-3p, glioma, neuronal pentraxin 1, insulin receptor substrate 1, phosphoinositide-3 kinase, AKT

\section{Introduction}

Gliomas are the most prevalent type of malignant brain tumor, accounting for $46-70 \%$ of all central nervous system (CNS) tumors, and have a devastating impact on the human health and life (1). The major characteristics of gliomas are their invasive growth and high rate of recurrence after intracranial operation, resulting in a poor prognosis of affected patients $(2,3)$. Gliomas have been classified by the World Health Organization into four grades: Grades I and II are for low-grade tumors, with Grade I tumors being benign tumors and Grade II tumors having the ability to recur as high-grade gliomas. Grade III and IV gliomas are high-grade tumors, of which Grade III gliomas tend to metastasize and recur, and Grade IV tumors are the most malignant gliomas (4). In spite of significant advances in surgical techniques and chemotherapeutic treatments, the median survival rate of glioma patients remains poor (5-7). It has been reported that the 5-year survival rate of patients with Grade I and II glioma is $30-70 \%$, while it is only 9-12 months for patients with Grade III and IV glioma (8). Therefore, it is of great importance to explore the molecular mechanisms underlying the development of gliomas and to identify novel biomarkers for predicting the progression of gliomas and identifying approaches for targeted therapies.

MicroRNAs (miRNAs/miRs) are a class of non-coding, single-stranded, endogenous small RNA molecules of 21-23 nucleotides in length, which regulate gene expression by binding to the 3 '-untranslated region (3'-UTR) of an mRNA target to promote mRNA degradation and/or translational repression $(9,10)$. It has been reported that miRNAs are involved in the modulation of a variety of biological processes, including cell proliferation, differentiation, apoptosis, migration and tumorigenesis $(11,12)$. miR-128-3p was identified to speed up cell cycle arrest and chromosomal instability in mitomycin C-treated lung cancer cells by suppressing spectrin alpha, non-erythrocytic 1 (13). It was also indicated that miR-128-3p is intimately associated with hepatocellular carcinoma $(14,15)$ and acute lymphoblastic leukemia (16). However, to date, the association between miR-128-3p and gliomas has remained to be determined in combination with a clinical and fundamental study.

In the present study, a total of 61 pairs of tumor tissues and paratumor tissues were collected from glioma patients 
and it was identified that the relative expression of miR-218-5p was downregulated, while neuronal pentraxin 1 (NPTX1) was upregulated in the tumor tissues. Through a bioinformatics analysis using Targetscan, NPTX1 was identified as a potential target of miR-218-5p, which was confirmed using a luciferase reporter assay. After the human glioma cells were transfected with miR-218-5p mimics or inhibitor, the insulin receptor substrate 1 (IRS-1)/phosphoinositide-3 kinase (PI3K)/AKT signaling pathway was suppressed or promoted, respectively. Furthermore, the IRS-1/PI3K/AKT signaling pathway was inhibited after NPTX1 was suppressed. Thus, miR-128-3p regulates glioma development via the IRS-1/PI3K/AKT signaling pathway by targeting NPTX1.

\section{Materials and methods}

Patients and tissue samples. Between March 2009 and April 2016, a total of 61 pairs of primary glioma and matched paratumor tissue samples were collected at the Department of Neurosurgery of the First Hospital of Lanzhou University (Lanzhou, China). The tumor and adjacent normal tissues were rapidly frozen in liquid nitrogen and preserved at $-70^{\circ} \mathrm{C}$ until use. None of the patients had received any blood transfusion, chemotherapy or radiotherapy prior to the surgery. Written informed consent was obtained from each patient and approval of the Ethics Committee of the First Hospital of Lanzhou University was obtained for using their tissue samples in the present study.

U251 cell culture and transfection. The U251 human glioma cell line was purchased from the cell bank of the Chinese Academy of Sciences (Shanghai, China) and was cultured in minimum essential medium/Earle's balanced salt solution (Hyclone; GE Healthcare, Little Chalfont, UK) supplemented with $10 \%$ fetal bovine serum (Hyclone; GE Healthcare), $50 \mathrm{U} / \mathrm{ml}$ penicillin and $50 \mathrm{mg} / \mathrm{ml}$ streptomycin at $37^{\circ} \mathrm{C}$ in a humidified atmosphere containing $5 \% \mathrm{CO}_{2}$. The cells were seeded in a 6 -well plate at $5 \times 10^{5}$ cells/well and the medium was replaced every 3 days until the confluency reached $\sim 70 \%$. miR-128-3p mimics (100 nM) or negative control (NC, nonsense miR); Sangon Biotech Co., Ltd., Shanghai, China) were transfected into U251 cells using Lipofectamine ${ }^{\circledR} 2000$ (Invitrogen; Thermo Fisher Scientific, Inc., Waltham, MA, USA) according to the manufacturer's protocol. After 4-6 $\mathrm{h}$ of transfection, the medium was replaced with fresh normal medium and $24 \mathrm{~h}$ later, total RNA or protein was extracted. In U251 cells, 100 nM NPTX1 small interfering (si)RNA reagent (Sangon Biotech Co., Ltd.) and its negative control were transfected into the cells. After $6 \mathrm{~h}$, the medium was replaced with fresh complete medium. Total protein and RNA were extracted $24 \mathrm{~h}$ later. The expression of the NPTX1 protein was detected by western blot analysis.

Luciferase activity assay. A fragment including the 3'-UTR of human NPTX1 was amplified from human genomic mRNA (NCBI Reference Sequence: NM_002522.3) using the following primer pair: 5'-AGCACTGCGCTAGACACTAGG GATAGAGTTGTAAAAAACACA-3' and 5'-GTGCAA ATAACTAGATTTTATATATATATTATATAGAACTGT-3'. pGL3-NPTX1-wild type (WT) reporter vector was constructed by interception of polymerase chain reaction (PCR) products of the 3'UTR of NPTX1, which was cut with XbaI and cloned into the corresponding sites of the pGL3 vector. The QuikChange II Site-Directed Mutagenesis kit (Takara Bio Inc., Dalian, China) was used to generate a mutation in the putative site of miR-128-5p recognition according to the manufacturer's protocol. A total of $400 \mathrm{ng}$ WT or mutant luciferase reporter, $100 \mathrm{nM}$ miRNA-128-3p mimics or their NC and $30 \mathrm{ng}$ pRL-TK Renilla luciferase reporter vector (Promega Corp., Madison, WI, USA) was added to each well of a 24-well plate containing $4-5 \times 10^{4}$ cells per well. After transfection for $48 \mathrm{~h}$, cells were collected and luciferase activity was measured using the Dual-Luciferase ${ }^{\circledR}$ Reporter Assay system (Promega Corp.).

Bioinformatics analysis. The Targetscan online platform (http://www.targetscan.org/) was used to predict the target genes of miR-128-5p with reference to the human gene sequence. The predicted target genes were denoted and further analysis was performed to verify them. Furthermore, the specific binding sequence of the 3'UTR of NPTX1 was also predicted with this software.

Reverse transcription-quantitative (RT-q) PCR analysis. Total RNA was extracted from tissues of glioma or transfected cells using TRIzol reagent (Invitrogen; Thermo Fisher Scientific, Inc.), and $1 \mu \mathrm{g}$ total RNA was reverse transcribed using a PrimeScript RT Reagent kit with a gDNA Eraser (cat. nos. RR047A and RR047B; each, Takara Bio, Inc., Dalian, China) according to the manufacturer's protocol. Subsequently, PCR amplification was performed in a total reaction volume of $20 \mu \mathrm{l}$ comprising $1 \mu \mathrm{l}$ NPTX1 primers (0.2 $\mu \mathrm{mol} / 1$, Sangon Biotech Co., Ltd.), $10 \mu \mathrm{l} \mathrm{SYBR}$ green (Takara Bio, Inc.), $6 \mu 1$ diethyl pyrocarbonate-treated water (Sangon Biotech Co., Ltd.) and $2 \mu \mathrm{l}$ complementary DNA product, in a Real-Time LightCycler ${ }^{\circledR} 96$ System (Roche Diagnostics, Basel, Switzerland). The sequences of the primers were as follows: miR-128-3p forward, 5'-GACTGC CGAGCGAGCG-3' and reverse, 5'-GACGCCGAGGCACTC TCTCCT-3'; U6 forward, 5'-CCATCGGAAGCTCGTATA CGAAATT-3' and reverse, 5'-GGCCTCTCGAACTTGCGT GTCAG-3'; NPTX1 forward, 5'-TTGACAGTTGCATCA CAACG-3' and reverse, 5'-CCAGCTATGGCCTGCGAC CG-3'; $\beta$-actin forward, GCTGTCCCTGTATGCCTCT-3' and reverse, TGTCACGCACGATTTCC-3'. The following thermocycling conditions were applied: $5 \mathrm{sec}$ at $95^{\circ} \mathrm{C}$, followed by 40 cycles of $1 \mathrm{sec}$ at $95^{\circ} \mathrm{C}$ and $20 \mathrm{sec}$ at $65^{\circ} \mathrm{C}$, followed by $5 \mathrm{sec}$ at $72^{\circ} \mathrm{C}$. The comparative $2^{-\Delta \Delta \mathrm{Cq}}$ method was used for relative quantification of gene expression (17). Each sample utilized in reverse transcription and the PCR amplification of cDNA were analyzed three times.

Western blot analysis. Total protein was extracted from collected tissues and cells using radioimmunoprecipitation assay lysis buffer (Beyotime Institute of Biotechnology, Haimen, China) supplemented with $100 \mathrm{X}$ proteinase inhibitor and phosphatase inhibitor, and then the proteins $(25 \mu \mathrm{g})$ were separated by $10 \%$ SDS-PAGE following protein determination using the BCA method. Subsequently, the proteins were transferred to a polyvinylidene difluoride membrane (EMD Millipore, Billerica, MA, USA), which was then blocked with $5 \%$ non-fat milk for $1 \mathrm{~h}$ at room temperature. The primary 
antibodies were anti-NPTX1 (cat. no. 3725; 1:1,000 dilution), anti-IRS-1 (cat. no. 2382; 1:500 dilution), anti-phosphorylated (p)-IRS-1 (Tyr895) (cat. no. 3070; 1:500 dilution), anti-PI3K (cat. no. 4292; 1:1,000 dilution) anti-p-AKT (ser473; cat. no. $12694 ; 1: 1,000$ dilution), anti-AKT (cat. no. 2938; 1:1,000 dilution) and anti- $\beta$-actin (cat. no. 4970; 1:1,000 dilution) and were all rabbit monoclonal antibodies (Cell Signaling Technology, Inc., Danvers, MA, USA) in $4^{\circ} \mathrm{C}$ overnight. The secondary antibody (cat. no. A0208; Beyotime Institute of Biotechnology) was used at the dilution of 1:5,000 and incubated at room temperature for $90 \mathrm{~min}$. Blots were visualized with an enhanced chemiluminescence kit (cat. no. 15159; Invitrogen; Thermo Fisher Scientific, Inc.). The gray bands were analyzed with Image J software 10.8 (National Institutes of Health, Bethesda, MD, USA) to compare the expression between targeted proteins and internal controls.

U251 proliferation assay. To detect the proliferation activity of transfected U251 cells, the MTT assay was performed using a Cell Proliferation Kit I (Sigma-Aldrich; Merck $\mathrm{KGaA}$, Darmstadt, Germany) in a 96-well plate at a density of $4 \times 10^{3}$ per well according to the manufacturer's instructions. After 24, 48 and $72 \mathrm{~h}$ of incubation, MTT-formazan production was estimated by a VersaMax (Molecular Devices, LLC, Sunnyvale, CA, USA) at $570 \mathrm{~nm}$ to evaluate the proliferation rate. The index was determined at 48 and $72 \mathrm{~h}$ and normalized to that at $24 \mathrm{~h}$.

Statistical analysis. Values are expressed as the mean \pm standard error of the mean. All statistical analyses were performed using SPSS 17.0 software (SPSS, Inc., Chicago, IL, USA). The differences between two groups were compared using a paired Student's t-test and the differences between three or more groups using one-way analysis of variance followed by a Tukey's post-hoc test. $\mathrm{P}<0.05$ was considered to indicate a statistically significant difference.

\section{Results}

miR-128-3p and NPTX1 are differentially expressed in glioma tissues. The Targetscan search indicated that NPTX1 was a target gene of miR-128-3p. Thus, miR-128-3p and NPTX1 was assessed in glioma tissues. Between March 2009 and April 2016, a total of 61 pairs of glioma tumor tissues and the adjacent normal tissues were collected. RT-qPCR analysis indicated that the relative expression of miR-128-3p was significantly decreased, while NPTX1 was elevated in the tumor tissues compared with that in the normal tissues (Fig. 1A and B). In addition, the relative expression of miR-128-3p was also lower in metastatic tissues than that in non-metastatic tissues, but the NPTX1 expression was higher in metastatic tissues (Fig. 1C and D). According to the diagnostic criteria of the world health organization, the glioma patients were stratified according to their grade [grade I $(n=22)$, II $(n=20)$ and III+IV ( $=19)]$. The miR-128-3p expression was lowest in the grade III+IV group but highest in the grade I group. Conversely, NPTX1 expression was the highest in the grade III+IV group, while it was lowest in the grade I group (Fig. 1E and F). These results illustrated that miR-128-3p and NPTX1 are closely associated with gliomas.
NPTX1 is targeted by miR-128-3p. In order to further clarify the association between miR-128-3p and NPTX1, a Targetscan software analysis was performed to identify the miR-128-3p binding sequence in the 3 '-UTR of NPTX1 and the core of the binding sequence was deleted to generate a mutant sequence (Fig. 2A and B). Subsequently, U251 cells were co-transfected with miR-128-3p mimics and luciferase reporter vectors containing the WT or mutant miR-128-3p binding sequence of the 3'-UTR of NPTX1. It was revealed that the luciferase activity was significantly decreased in the WT group (NPTX1 $\mathrm{WT}+\mathrm{miR}-128-3 \mathrm{p}$ mimic group) in comparison with the mutant (NPTX1 mutant + miR-128-3p mimic group) and blank group (Blank + miR-128-3p mimic group), while no difference was observed between the mutant and blank groups (Fig. 2C). Of note, the luciferase activity was upregulated after the reporter vector driven by the binding sequence in the 3'-UTR of NPTX1 and miR-128-3p inhibitor (NPTX1 3'-UTR + miR-128-3p inhibitor group) were co-transfected into the U251 cells compared with the blank group (NPTX1 3'-UTR + Blank). Furthermore, the opposite result was obtained after the reporter vector driven by the binding sequence in the 3'-UTR of NPTX1 and miR-128-3p mimics (NPTX1 3'-UTR + miR-128-3p mimic group) were transfected into the cells in comparison with the blank group (Fig. 2D). In U251 cells transfected with miR-128-3p mimics, the expression of NPTX1 was decreased at the protein level (Fig. 2E), while the opposite effect was obtained after miR-128-3p inhibitor was transfected into the cells (Fig. 2F).

miR-128-3p mimics suppress IRS-1/PI3K/AKT signaling in U251 cells. Cultured U251 cells were transfected with miR-128-3p mimics or NC. Through visual observation, it was determined that the proliferation rate of the cells was reduced in the miR-128-3p mimics group (Fig. 3A) in comparison with that in the NC group (Fig. 3B). An MTT assay confirmed that the proliferation rate of the cells in the miR-128-3p mimics group was significantly lower than that in the NC group (Fig. 3C). In addition, western blot analysis clearly proved that compared with those in the NC group, the protein levels of p-IRS-1, PI3K and p-AKT were significantly decreased in the miR-128-3p mimics group, while the expression of IRS-1 and AKT was significantly increased (Fig. 3D and E). This result indicated that the IRS-1/PI3K/AKT signaling pathway was suppressed by the miR-128-3p mimics.

miR-128-3p inhibitor increases IRS-1/PI3K/AKT signaling in U251 cells. miR-128-3p inhibitor and its NC were transfected into the cultured U251 cells, and visual observation revealed that the proliferation rate of the cells was increased in the miR-128-3p inhibitor group (Fig. 4A) in comparison with that in the NC group (Fig. 4B). An MTT assay confirmed that the proliferation rate of the cells in the miR-128-3p inhibitor group was higher than that in the NC group (Fig. 4C). Furthermore, compared with those in the NC group, the levels of p-IRS-1, PI3K and p-AKT were significantly increased in the miR-128-3p inhibitor group, while the expression of IRS-1 and AKT was not significantly different (Fig. 4D and E). This result indicated that IRS-1/PI3K/AKT signaling was enhanced after miR-128-3p was inhibited. 
A

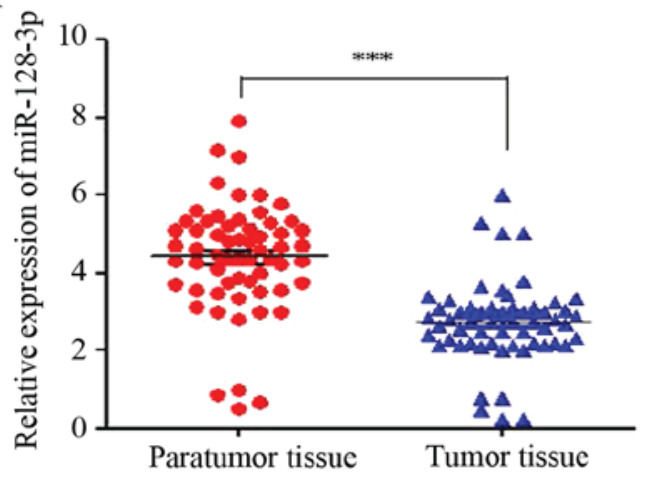

$\mathrm{C}$

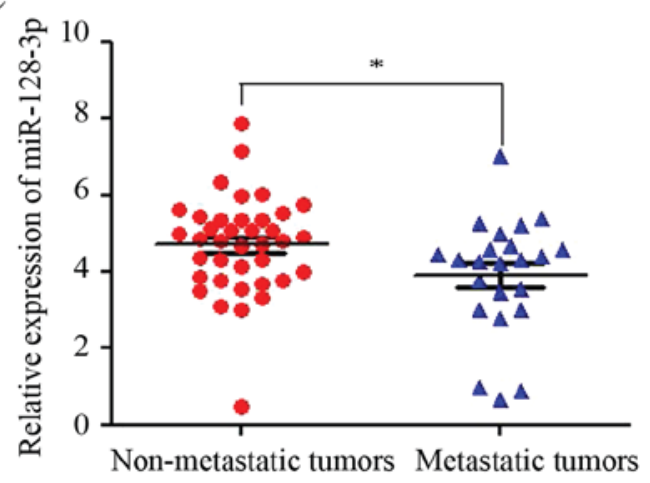

E

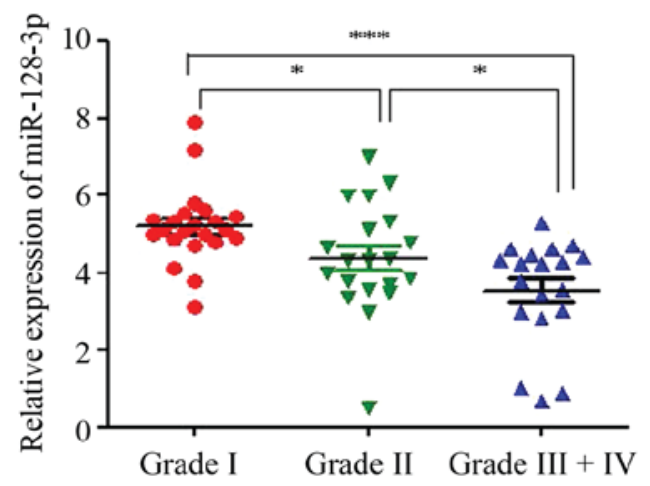

$\mathrm{B}$

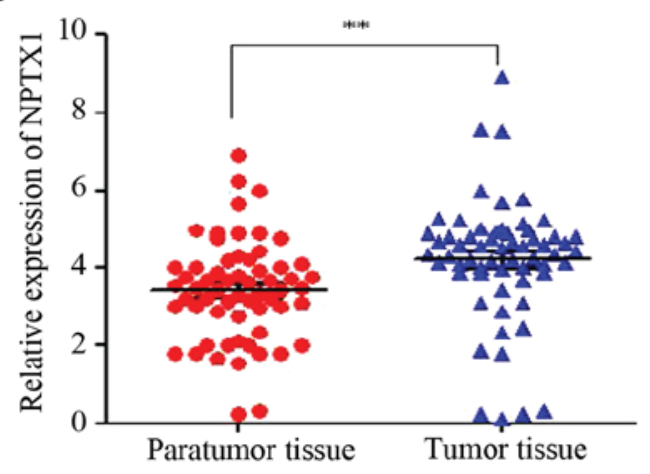

$\mathrm{D}$

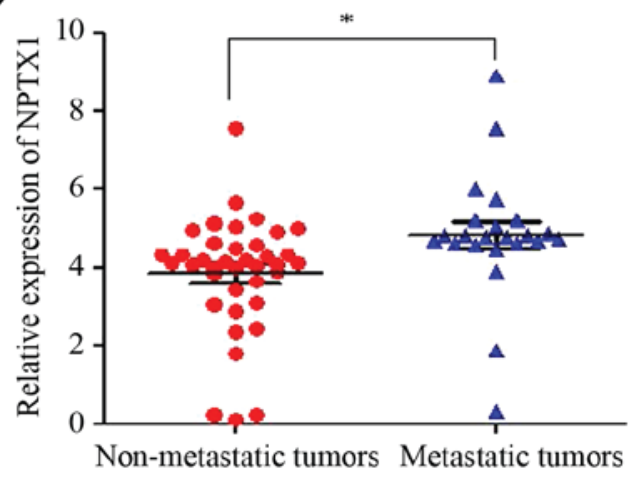

$\mathrm{F}$

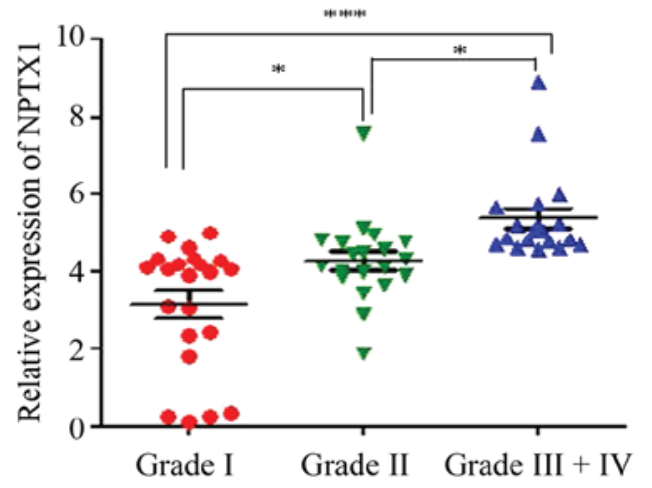

Figure 1. Differential expression of miR-128-3p and NPTX1 in glioma, adjacent normal and metastatic tissues. (A) The relative expression of miR-128-3p was significantly decreased in tumor tissues. (B) NPTX1 expression was obviously elevated in tumor tissues. (C) In metastatic tumor tissues, the expression of miR-128-3p was lower than that in non-metastatic tumor tissues. (D) The expression of NPTX1 was significantly promoted in metastatic tumor tissues in comparison with that in non-metastatic tumor tissues. (E) The expression of miR-128-3p in grade III and IV was significantly decreased compared with that in grade I and II glioma tissues, but it was lower in grade II than in grade I glioma tissues. (F) The expression of NPTX1 was notably increased in grade III and IV glioma compared with that in grade I and II glioma, but it was higher in grade II than in grade I. ${ }^{*} \mathrm{P}<0.05$, ${ }^{* *} \mathrm{P}<0.01,{ }^{* * * *} \mathrm{P}<0.001$. NPTX1, neuronal pentraxin 1 ; miR, microRNA.

Suppression of NPTX1 inhibits IRS-1/PI3K/AKT signaling. In order to further demonstrate the association between NPTX1 and the IRS-1/PI3K/AKT signaling pathway, NPTX1 siRNA was transfected into the cultured U251 cells, which caused a significant decrease in the levels of p-IRS-1, PI3K and p-AKT compared with that in the NC group (Fig. 5A and B). However, the expression of total IRS-1 and AKT was increased in the NPTX1 siRNA group. This meant that compared with the protein levels normalized to $\beta$-actin, the p-IRS-1 and p-AKT levels relative to IRS-1 and AKT, respectively, were even higher. However, after the expression of NPTX1 was inhibited with siRNA, the expression of miR-128-3p was not significantly different (Fig. 5C).

\section{Discussion}

Glioma is the most prevalent type of malignant carcinoma of the CNS and has a yearly incidence of almost 6 per 100,000 individuals in the US (18). Gliomas represent a severe treat to human health and life, and are therefore intensively studied. During glioma cell migration and invasion, transforming growth factor- $\beta$ receptor type 2 exerts a key role and it may 
A

\begin{tabular}{|c|c|c|}
\hline & $\begin{array}{c}\text { Predicted consequential pairing of target region (top) and } \\
\text { miRNA (bottom) }\end{array}$ & $\begin{array}{l}\text { Site } \\
\text { type }\end{array}$ \\
\hline $\begin{array}{l}\text { Position } 2624-2630 \text { of NPTX1 } 3 \text { ' UTR } \\
\text { hsa-miR-128-3p }\end{array}$ & $\begin{array}{l}\text { 5, ...GCCCCUCGACUCCCUCACUGUGU.... } \\
\text { 3, } 1111 \text { UUUCUCUGGCCAAGUGACACU }\end{array}$ & $\begin{array}{c}7 \mathrm{mer}- \\
\mathrm{m} 8\end{array}$ \\
\hline
\end{tabular}

B

WT: CUGAGCCAAGCCCCUCGACUCCCCACUGUGUUGACACUUGGCACUUU

Mutant: CUGAGCCAAGCCCCUCGACUCCC -------- UUGACACUUGGCACUUU

$\mathrm{C}$

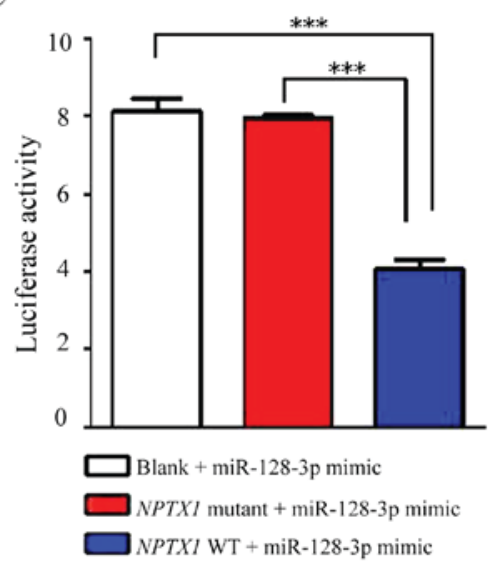

E
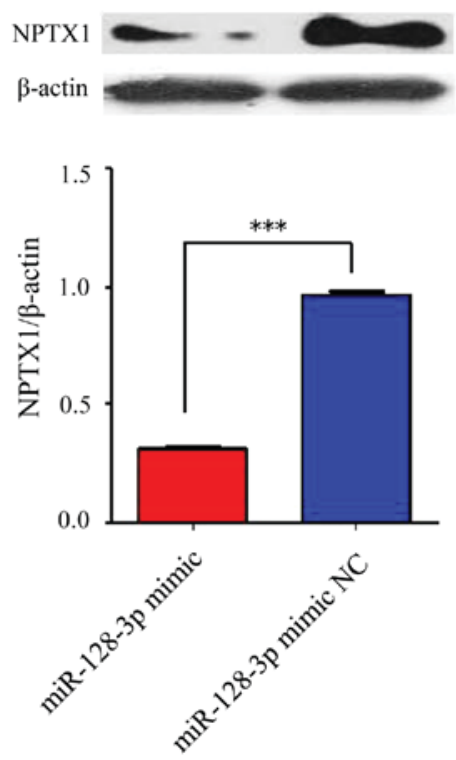

$\mathrm{D}$

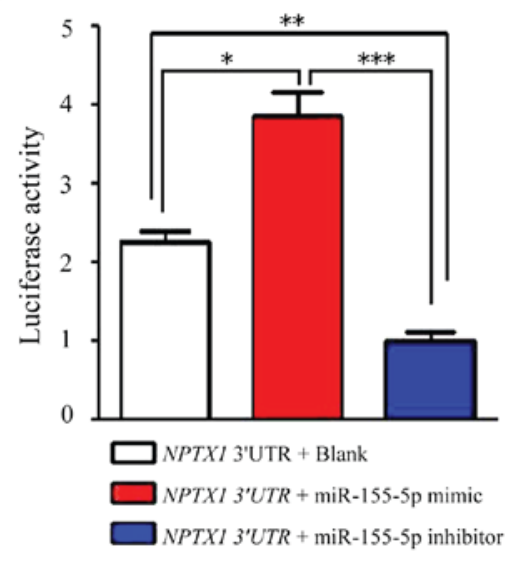

F
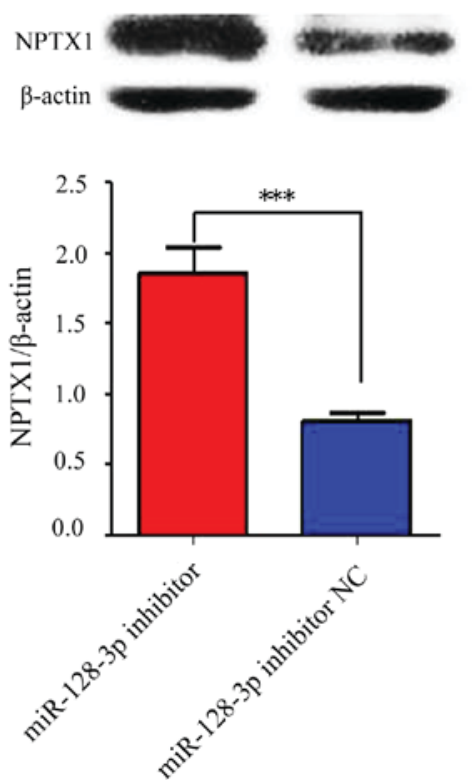

Figure 2. NFTX-1 is a direct target of miR-128-3p. (A) The predicted binding sequences of the 3'-UTR of NPTX1 and miR-128-3p. (B) The sequences on the top refer to the complete binding sequences of the 3'-UTR of NPTX1 and miR-128-3p, while the ones at the bottom refer to the mutation sequences without core sequences. (C) After the binding sequences of 3'-UTR of NPTX1 and miR-128-3p mimics were transfected into U251 cells, the luciferase activity was obviously decreased; however, no statistically significant difference was identified after their mutated sequences were transfected into the cells. (D) The luciferase activity was significantly promoted after the binding sequences of NPTX1 3'-UTR and miR-128-3p inhibitor were transfected into U251 cells, but the results were opposite after the binding sequences of the NPTX1 3'-UTR and miR-128-3p mimics were transfected into the cells. (E and F) After U251 cells were transfected with miR-128-3p mimics, the protein expression of NPTX1 was decreased compared with that in the NC group, while it was increased after transfection with miR-128-3p inhibitor. ${ }^{*} \mathrm{P}<0.05,{ }^{* *} \mathrm{P}<0.01,{ }^{* * *} \mathrm{P}<0.001$. NPTX1, neuronal pentraxin 1; miR, microRNA; UTR, untranslated region; WT, wild-type; NC, negative control; hsa, Homo sapiens.

serve as a prognostic predictor and a therapeutic target for glioma (19). It has been indicated that sea buckthorn leaf extract suppresses the proliferation of rat glioma cells via inducing early events of apoptosis and it may therefore serve as a potential therapeutic for the treatment of glioma (20). It has been reported that atorvastatin has cytotoxic effects and 
A

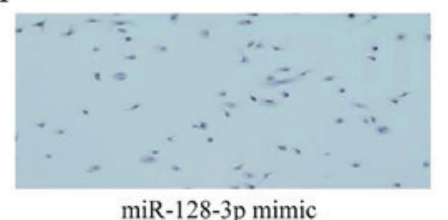

B

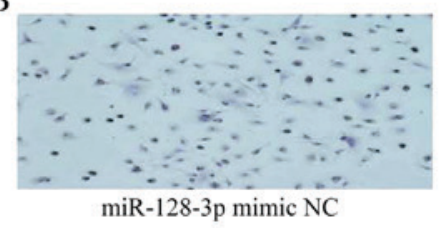

$\mathrm{C}$

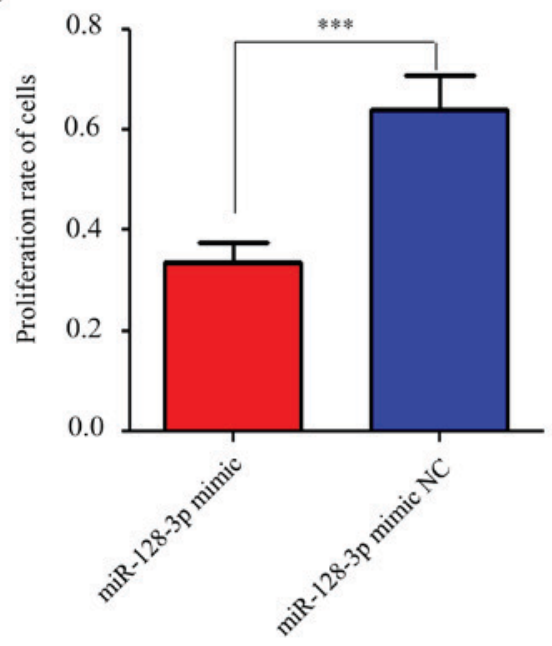

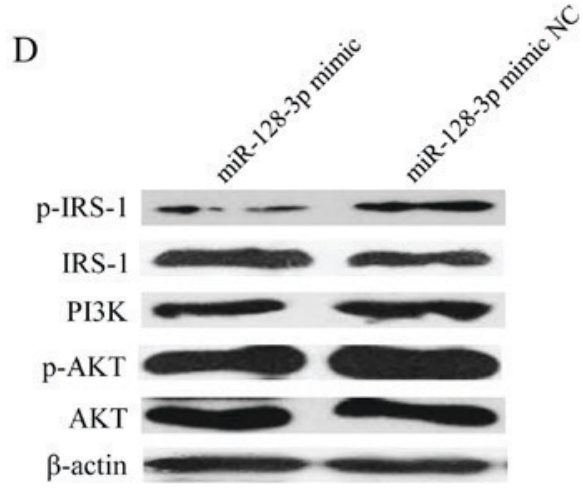

$\mathrm{F}$

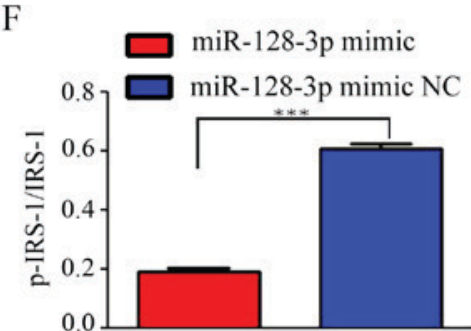

$\mathrm{E}$

$\square$ miR-128-3p mimic

$\square$ miR-128-3p mimic NC

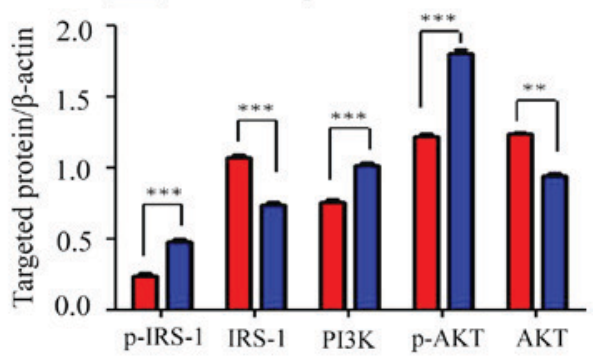

G

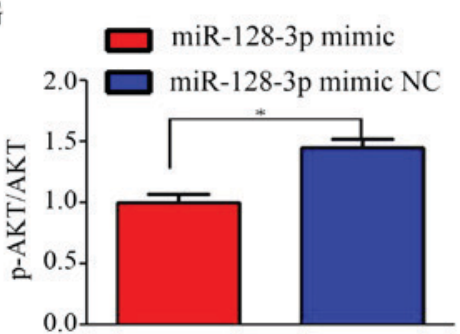

Figure 3. miR-128-3p mimics suppress the IRS-1/PI3K/AKT pathway. (A and B) The amount of U251 cells was after transfection with miR-128-3p mimics as compared with that in the NC group (magnification, x200 magnification). (C) An MTT assay revealed that the proliferation rate of the cells was statistically decreased in the miR-128-3p mimics group in comparison with that in the NC group. (D and E) Western blot analysis indicated that the levels of p-IRS-1, PI3K and p-AKT in the miR-128-3p mimics group were significantly decreased compared with those in the NC group. Furthermore, the expression of IRS-1 and AKT in the miR-128-3p mimics group was higher than that in the NC group. The ratio of (F) p-IRS-1/IRS-1 and (G) $p-A K T / A K T . ~{ }^{*} \mathrm{P}<0.05,{ }^{* *} \mathrm{P}<0.01$, ${ }_{* * * *} \mathrm{P}<0.001$. p-IRS-1, phosphorylated insulin receptor substrate 1; PI3K, phosphoinositide-3 kinase; miR, microRNA; NC, negative control.

induces autophagy, and simultaneously reduces the migration and proliferation of human A172 glioma cells (21). However, in spite of the abundancy of studies on gliomas, the exact molecular mechanisms associated with gliomas and therapeutic targets leading to an efficient treatment approach remain to be elucidated, and additional study is therefore required.

Certain miRNAs are known to be associated with tumor development and progression through regulating the translation or causing degradation of their target mRNAs $(22,23)$. Regarding the elucidation of the exact mechanisms underlying the pathological processes of various diseases, particularly cancers, miRNAs may provide a novel approach for their diagnosis and treatment (24). A variety of studies revealed that
miRNAs are involved in the development and progression of gliomas. A previous study suggested that low expression of miR-508-5p was associated with glioma patient survival; it may therefore serve as a novel prognostic factor and also lead to novel treatment strategies for glioma (25). It has been proved that overexpression of miR-497 is associated with resistance to temozolomide in human glioma cells by targeting mammalian target of rapamycin/B-cell lymphoma 2 (26). It has also been reported that miR-302a is an important tumor suppressor of glioma progression by directly targeting GRB2-associated binding protein, thus providing novel insight into the molecular mechanisms underlying the genesis, development and progression of glioma (27). Furthermore, miR-128-3p was reported 
A

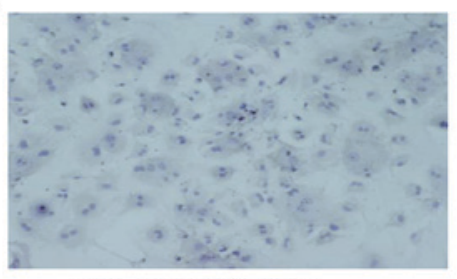

miR-128-3p inhibitor

B

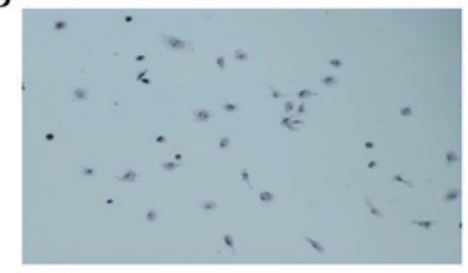

miR-128-3p inhibitor NC

D

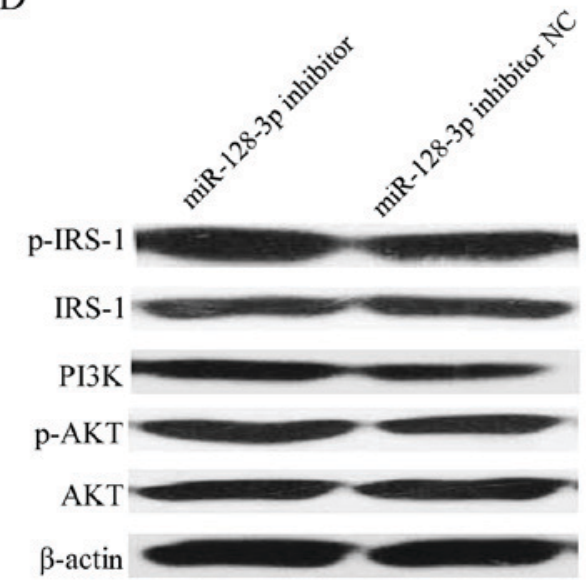

C

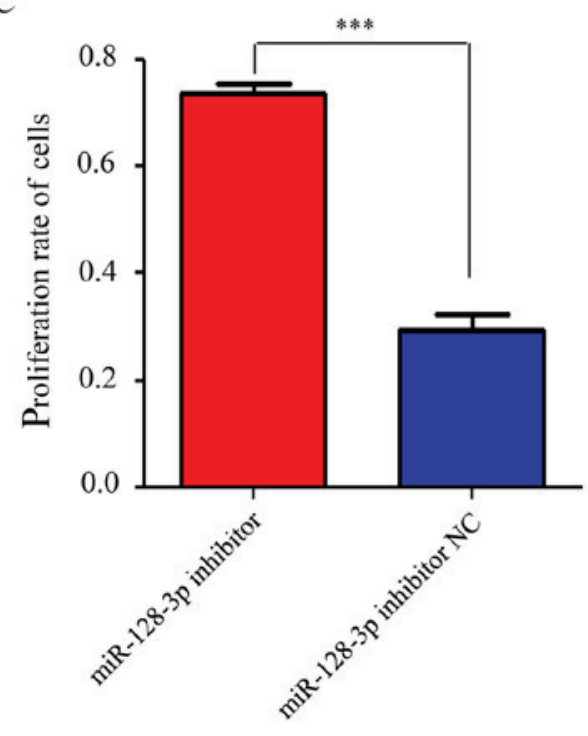

E
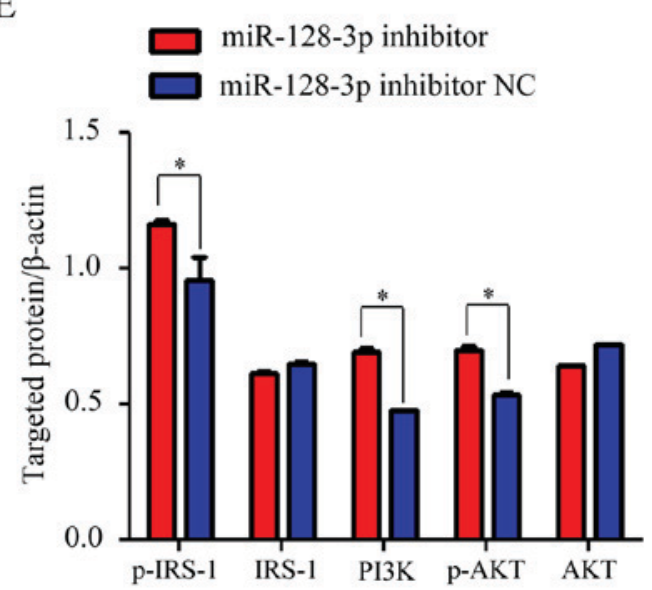

Figure 4. IRS-1/PI3K/AKT signaling is activated after inhibition of miR-128-3p. (A and B) Following transfection with miR-128-3p inhibitor, the amount of U251 cells was increased compared with that in the NC group. (C) An MTT assay indicated that the proliferation rate of the cells in the miR-128-3p inhibitor group was significantly elevated in comparison with that in the NC group. (D and E) Western blot analysis indicated that the levels of p-IRS-1, PI3K and p-AKT in the miR-128-3p inhibitor group were significantly higher than those in the NC group. In addition, the expression of IRS-1 and AKT was not significantly different between the miR-128-3p inhibitor group and the NC group. $\mathrm{P}<0.05,{ }^{* * * *} \mathrm{P}<0.001$. p-IRS-1, phosphorylated insulin receptor substrate 1 ; PI3K, phosphoinositide-3 kinase; miR, microRNA; NC, negative control.

to be closely associated with lung cancer (13), hepatocellular carcinoma $(14,15)$ and gastric cancer $(28)$. However, the association between miR-128-3p and glioma has remained to be determined.

The present study indicated that the expression of miR-128-3p was significantly lower in glioma tissues than in paratumor tissues. In addition, in metastatic tissues, the relative expression of miR-128-3p was also decreased in comparison with that in non-metastatic tumor tissues. Furthermore, the relative expression of $\mathrm{miR}-128-3 \mathrm{p}$ was lowest in grade III+IV glioma, followed by in that in grade II and grade I glioma. These results demonstrated that miR-128-3p was intimately associated with gliomas. It has been demonstrated that a synergistic action of metformin and aspirin regulates the transcriptional profile of pancreatic cancer cells and NPTX1 was upregulated by $>10$-fold (29). Specific gene expression signatures have been identified in individuals and NPTX1 expression was decreased in mouse strains susceptible to pulmonary adenomas (30). NPTX1 was reported to be intimately associated with colorectal cancer (31), high-grade cervical intraepithelial neoplasia and cervical cancer (32) as biomarkers involved in methylation. These previous studies demonstrated that NPTX1 is closely associated with a variety of tumor types, but the association between NPTX1 and glioma has remained to be elucidated. In the present study, the expression of NPTX1 was significantly elevated in tumor tissues compared with that in normal tissues. In metastatic tissues, the relative expression of NPTX1 was also elevated in comparison with that in non-metastatic tumor tissues. In addition, the relative expression of NPTX1 was the highest in grade III $+\mathrm{IV}$, followed by that in grade II and grade I glioma. These results clearly illustrated that NPTX1 was 
A

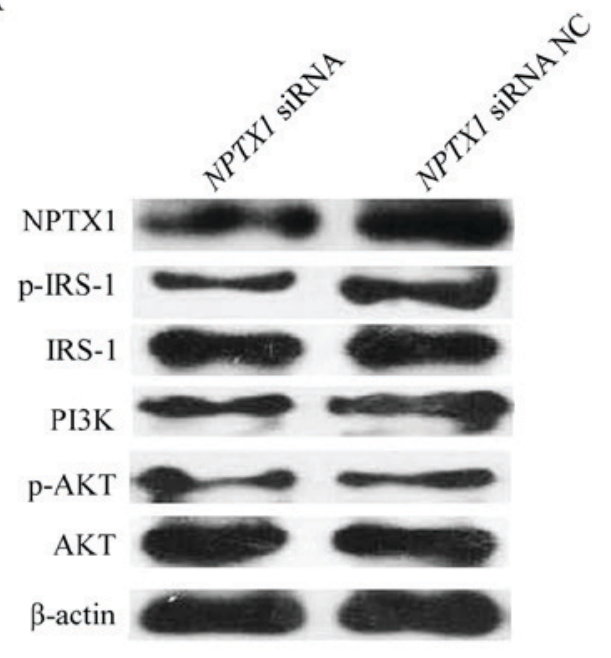

B

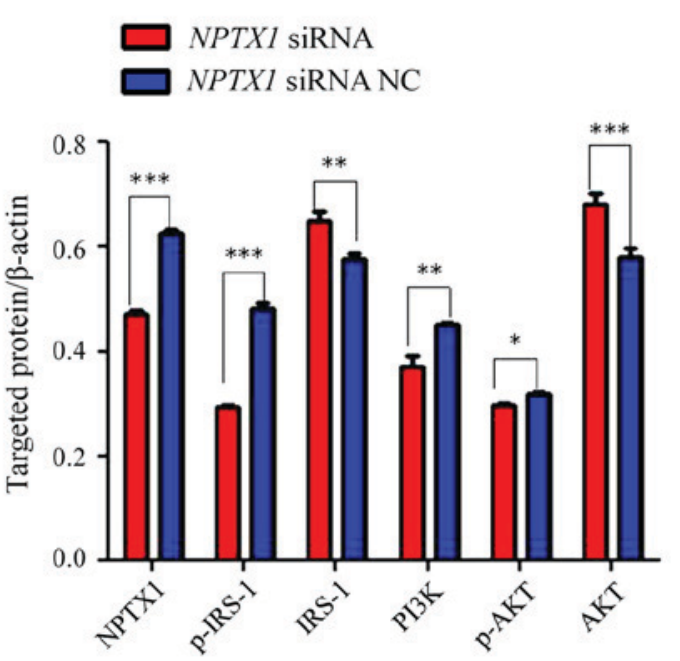

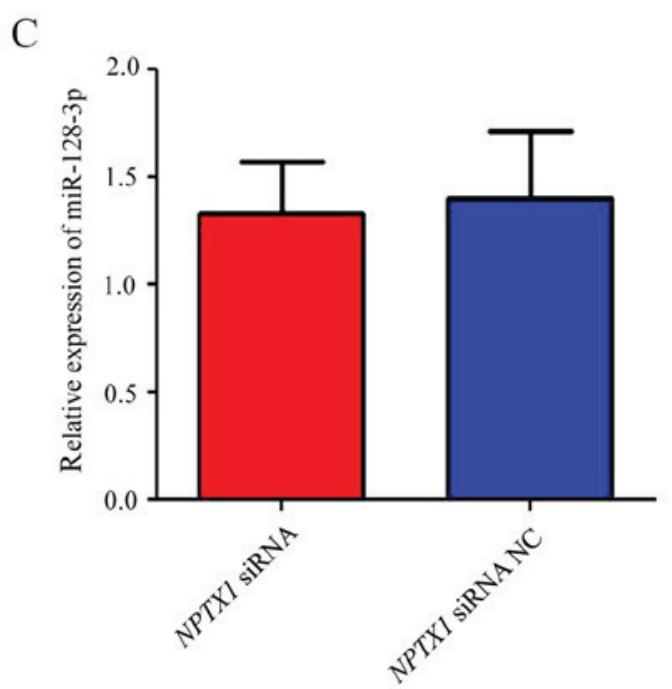

Figure 5. IRS-1/PI3K/AKT signaling pathway is suppressed by inhibition of NPTX1. (A and B) The expression of p-IRS-1, PI3K and p-AKT was significantly suppressed after transfection with NPTX1 siRNA, which inhibited the expression of NPTX1 at the translational level. However, the expression of IRS-1 and AKT was significantly increased in the NPTX1 siRNA group in comparison with that in the NC group. (C) After NPTX1 siRNA was transfected into the cultured U251 cells, the expression of miR-128-3p in the NPTX1 siRNA group was not significantly different from that in the NC group. "P<0.05, **P<0.01, ${ }^{* * *} \mathrm{P}<0.001$. NPTX1, neuronal pentraxin 1; miR, microRNA; siRNA, small hairpin RNA; NC, negative control.

positively associated with glioma and inversely associated with miR-128-3p expression. A Targetscan analysis, which initially confirmed that miR-128-3p could target NPTX1, followed by a luciferase reporter assay and transfection experiments further confirmed this result. It has been demonstrated that leptin stimulates the migration of human prostate cancer cells, and that one of the underlying mechanisms was transcriptional upregulation of $\alpha \mathrm{v} \beta 3$ integrin expression through the [long form of the obese gene (leptin) receptor] OBR1/IRS-1/PI3K/Akt/nuclear factor (NF)- $\kappa B$ signal transduction pathway (33). In addition, leptin promotes the migration of chondrosarcoma cells by increasing $\alpha v \beta 3$ integrin expression through the OBR1/IRS-1/PI3K/Akt/NF- $\mathrm{KB}$ signal transduction pathway (34). A previous study suggested that the IRS-1/PI3K/AKT signaling pathway was positively correlated with tumor growth, proliferation and migration. In the present study, after the glioma cells were transfected with miR-128-3p mimics, the IRS-1/PI3K/AKT signaling pathway was inhibited, while it was activated by transfection with miR-128-3p inhibitor. In addition, after NPTX1 was suppressed with specific siRNA, the IRS-1/PI3K/AKT signaling pathway was inhibited; however, the expression of miR-128-3p was not affected. These results demonstrated that in the growth and differentiation of glioma cells, miR-128-3p regulates the activity of the NPTX1/IRS-1/PI3K/AKT signaling pathway. Furthermore, the IRS-1/PI3K/AKT signaling pathway was situated downstream of NPTX1, which was able to modulate IRS-1/PI3K/AKT signaling pathway activity. However, miR-128-3p could not be reversely regulated by NPTX1 as expected, which was directly targeted by miR-128-3p.

The present study provided novel biomarkers, miR-128-3p and NPTX1, for the diagnosis of glioma, which may also 
serve as therapeutic targets in the treatment of glioma. miR-128-3p was revealed to have a vital role in the regulation of glioma growth, proliferation through activating the IRS-1/PI3K/AKT signaling pathway by targeting NPTX1.

\section{Acknowledgements}

Not applicable.

\section{Funding}

No funding was received.

\section{Availability of data and materials}

The datasets used and/or analyzed during the current study are available from the corresponding author on reasonable request.

\section{Authors' contributions}

QLG was fully responsible for the study design, experimental adjustment and drafting and finalizing the manuscript. LMH and BW performed the majority of the experiments. MHZ and YHZ performed the transfection assays and certain protein measurements by western blotting and statistical analysis. JGX and GY conducted the densitometry, statistical analysis and participated in the coordination of the manuscript. All authors read and approved the final manuscript.

\section{Ethics approval and consent to participate}

The study was approved by the Ethics Committee of the First Hospital of Lanzhou University (Lanzhou, China) and written informed consent was obtained from each patient.

\section{Patient consent for publication}

Not applicable.

\section{Competing interests}

The authors declare that they have no competing interests.

\section{References}

1. Qaddoumi I, Sultan I and Gajjar A: Outcome and prognostic features in pediatric gliomas: A review of 6212 cases from the surveillance, epidemiology, and end results database. Cancer 115 5761-5770, 2009.

2. McCarthy BJ, Shibui S, Kayama T, Miyaoka E, Narita Y, Murakami M, Matsuda A, Matsuda T, Sobue T, Palis BE, et al: Primary CNS germ cell tumors in Japan and the United States: An analysis of 4 tumor registries. Neurol Oncol 14: 1194-1200, 2012.

3. Ye ZN, Liu JP, Wu LY, Zhang XS, Zhuang Z, Chen Q, Lu Y, Liu CG, Zhang ZH, Zhang HS, et al: Retraction notice to 'Downregulation of miR-204 expression correlates with poor clinical outcome of glioma patients' [Hum Pathol 2017; 63: 46-52]. Hum Pathol 63: R1, 2017.

4. Keller M, Rüegg A, Werner S and Beer HD: Active caspase-1 is a regulator of unconventional protein secretion. Cell 132: 818-831, 2008.

5. Berges R, Balzeau J, Peterson AC and Eyer J: A tubulin binding peptide targets glioma cells disrupting their microtubules, blocking migration, and inducing apoptosis. Mol Ther 20: $1367-1377,2012$
6. Li B, Huang MZ, Wang XQ, Tao BB, Zhong J, Wang XH, Zhang WC and Li ST: TMEM140 is associated with the prognosis of glioma by promoting cell viability and invasion. J Hematol Oncol 8: 89, 2015.

7. Wang Z, Guo Q, Wang R, Xu G, Li P, Sun Y, She X, Liu Q, Chen Q, Yu Z, et al: The D domain of LRRC4 anchors ERK1/2 in the cytoplasm and competitively inhibits MEK/ERK activation in glioma cells. J Hematol Oncol 9: 130, 2016.

8. Zhang X, Yang H, Gong B, Jiang C and Yang L: Combined gene expression and protein interaction analysis of dynamic modularity in glioma prognosis. J Neurooncol 107: 281-288, 2012.

9. Fabian MR and Sonenberg N: The mechanics of miRNA-mediated gene silencing: A look under the hood of miRISC. Nat Struct Mol Biol 6: 586-593, 2012.

10. Samir M, Vaas LA and Pessler F: MicroRNAs in the host response to viral infections of veterinary importance. Front Vet Sci 3: 86, 2016

11. Zhou W, Zou B, Liu L, Cui K, Gao J, Yuan S and Cong N: MicroRNA-98 acts as a tumor suppressor in hepatocellular carcinoma via targeting SALL4. Oncotarget 7: 74059-74073, 2016.

12. Choi E, Choi E and Hwang KC: MicroRNAs as novel regulators of stem cell fate. World J Stem Cells 5: 172-187, 2013.

13. Zhang R, Liu C, Niu Y, Jing Y, Zhang H, Wang J, Yang J, Zen K, Zhang J, Zhang CY and Li D: MicroRNA-128-3p regulates mitomycin C-induced DNA damage response in lung cancer cells through repressing SPTAN1. Oncotarget 8: 58098-58107, 2016.

14. Huang CY, Huang XP, Zhu JY, Chen ZG, Li XJ, Zhang XH, Huang S, He JB, Lian F, Zhao YN and Wu GB: miR-128-3p suppresses hepatocellular carcinoma proliferation by regulating PIK3R1 and is correlated with the prognosis of HCC patients. Oncol Rep 33: 2889-2898, 2015.

15. Yu D, Green B, Marrone A, Guo Y, Kadlubar S, Lin D, Fuscoe J, Pogribny I and Ning B: Suppression of CYP2C9 by microRNA hsa-miR-128-3p in human liver cells and association with hepatocellular carcinoma. Sci Rep 5: 8534, 2015.

16. Mets E, Van Peer G, Van der Meulen J, Boice M, Taghon T, Goossens S, Mestdagh P, Benoit Y, De Moerloose B, Van Roy N, et al: MicroRNA-128-3p is a novel oncomiR targeting PHF6 in T-cell acute lymphoblastic leukemia. Haematologica 99: 1326-1333, 2014.

17. Livak KJ and Schmittgen TD: Analysis of relative gene expression data using real-time quantitative PCR and the 2(-Delta Delta C(T)) method. Methods 25: 402-408, 2001.

18. Easwaran H, Tsai HC and Baylin SB: Cancer epigenetics: Tumor heterogeneity, plasticity of stem-like states, and drug resistance. Mol Cell 54: 716-727, 2014

19. Hu S, Chen H, Zhang Y, Wang C, Liu K, Wang H and Luo J: MicroRNA-520c inhibits glioma cell migration and invasion by the suppression of transforming growth factor- $\beta$ receptor type 2 . Oncol Rep 37: 1691-1697, 2017.

20. Kim SJ, Hwang E, Yi SS, Song KD, Lee HK, Heo TH, Park SK, Jung YJ and Jun HS: Sea buckthorn leaf extract inhibits glioma cell growth by reducing reactive oxygen species and promoting apoptosis. Appl Biochem Biotechnol 182: 1663-1674, 2017.

21. Oliveira KA, Dal-Cim T, Lopes FG, Ludka FK, Nedel CB and Tasca CI: Atorvastatin promotes cytotoxicity and reduces migration and proliferation of human A172Glioma cells. Mol Neurobiol 55: 1509-1523, 2018.

22. Dang X, Ma A, Yang L, Hu H, Zhu B, Shang D, Chen T and Luo Y: MicroRNA-26a regulates tumorigenic properties of EZH2 in human lung carcinoma cells. Cancer Genet 205: 113-123, 2012.

23. Qi P, Cheng SQ, Wang H, Li N, Chen YF and Gao CF: Serum microRNAs as biomarkers for hepatocellular carcinoma in Chinese patients with chronic hepatitis B virus infection. PLoS One 6: e28486, 2011

24. Shao XJ, Miao MH, Xue J, Xue J, Ji XQ and Zhu H: The down-regulation of microrna-497 contributes to cell growth and cisplatin resistance through PI3K/akt pathway in osteosarcoma. Cell Physiol Biochem 36: 2051-2062, 2015.

25. Liu YH, Li B, Meng FG and Qiu L: MiR-508-5p is a prognostic marker and inhibits cell proliferation and migration in glioma. Eur Rev Med Pharmacol Sci 21: 76-81, 2017.

26. Zhu D, Tu M,Zeng B, Cai L,Zheng W, SuZ and Yu Z: Up-regulation of miR-497 confers resistance to temozolomide in human glioma cells by targeting mTOR/Bcl-2. Cancer Med 6: 452-462, 2017.

27. Ma J, Yu J, Liu J, Yang X, Lou M, Liu J, Feng F, Ji P and Wang L: MicroRNA-302a targets GAB2 to suppress cell proliferation, migration and invasion of glioma. Oncol Rep 37: 1159-1167, 2017. 
28. Ibarrola-Villava M, Llorca-Cardeñosa MJ, Tarazona $\mathrm{N}$, Mongort C, Fleitas T, Perez-Fidalgo JA, Roselló S, Navarro S, Ribas G and Cervantes A: Deregulation of ARID1A, CDH1, cMET and PIK3CA and target-related microRNA expression in gastric cancer. Oncotarget 6: 26935-26945, 2015.

29. Yue W, Wang T, Zachariah E, Lin Y, Yang CS, Xu Q, DiPaola RS and Tan XL: Transcriptomic analysis of pancreatic cancer cells in response to metformin and aspirin: An implication of synergy. Sci Rep 5: 13390, 2015.

30. Stearns TM, Cario CL, Savage HS, Sundberg JP, Paigen B and Berndt A: Early gene expression differences in inbred mouse strains with susceptibility to pulmonary adenomas. Exp Mol Pathol 93: 455-461, 2012.

31. Mori Y, Olaru AV, Cheng Y, Agarwal R, Yang J, Luvsanjav D, Yu W, Selaru FM, Hutfless S, Lazarev M, et al: Novel candidate colorectal cancer biomarkers identified by methylation microarray-based scanning. Endocr Relat Cancer 18: 465-478, 2011.
32. Yang N, Eijsink JJ, Lendvai A, Volders HH, Klip H, Buikema HJ, van Hemel BM, Schuuring E, van der Zee AG and Wisman GB: Methylation markers for CCNA1 and C13ORF18 are strongly associated with high-grade cervical intraepithelial neoplasia and cervical cancer in cervical scrapings. Cancer Epidemiol Biomarkers Prev 18: 3000-3007, 2009.

33. Huang CY, Yu HS, Lai TY, Yeh YL, Su CC, Hsu HH, Tsai FJ, Tsai CH, Wu HC and Tang CH: Leptin increases motility and integrin up-regulation in human prostate cancer cells. J Cell Physiol 226: 1274-1282, 2011.

34. Yang SN, Chen HT, Tsou HK, Huang CY, Yang WH, Su CM, Fong YC, Tseng WP and Tang CH: Leptin enhances cell migration in human chondrosarcoma cells through OBRl leptin receptor. Carcinogenesis 30: 566-574, 2009.

This work is licensed under a Creative Commons Attribution-NonCommercial-NoDerivatives 4.0 International (CC BY-NC-ND 4.0) License. 